\title{
COVID-19 NAS PRISÕES: EFEITOS DA PANDEMIA SOBRE A SAÚDE MENTAL DE MULHERES PRIVADAS DE LIBERDADE
}

\author{
COVID-19 IN PRISONS: PANDEMIC EFFECTS ON THE \\ MENTAL HEALTH OF WOMEN DEPRIVED OF LIBERTY
}

\section{COVID-19 EN LAS PRISIONES: EFECTOS PANDÉMICOS EN LA SALUD MENTAL DE LAS MUJERES PRIVADAS DE LIBERTAD}

\author{
Gabriel da Cruz Santos ${ }^{1}$ \\ Tainá Cerqueira Simôa ${ }^{2}$ \\ Tânia Christiane Ferreira Bispo ${ }^{3}$ \\ Ridalva Dias Martins ${ }^{4}$ \\ Denise Santana Silva dos Santos ${ }^{5}$ \\ Aglaya Oliveira Lima Cordeiro de Almeida ${ }^{6}$
}

\begin{abstract}
Como citar este Artigo: Santos GC, Simôa TC, Bispo TCF, Martins RD, Santos DSS, Almeida AOLC. Covid-19 nas prisões: efeitos da pandemia sobre a saúde mental de mulheres privadas de liberdade. Rev baiana enferm. 2020;34:e38235.

Objetivo: investigar o autorrelato de sintomas de ansiedade relacionados à Covid-19 entre mulheres encarceradas. Método: estudo transversal, realizado em uma unidade prisional de Salvador, Bahia, no período de abril a maio de 2020. Os dados foram coletados por meio de questionário, sendo analisadas as frequências relativas e absolutas. Resultados: participaram 41 mulheres, majoritariamente jovens, de baixa escolaridade, mães solo e negras. 95\% da amostra relatou algum sintoma de ansiedade. Encontrou-se alta prevalência de preocupação consigo, com a segurança no presídio e com a família diante da pandemia. A maioria das mulheres encontrou mecanismos para atenuar os impactos psicológicos da pandemia. Conclusão: a fragilização dos vínculos familiares e a sensação de insegurança relacionada à Covid-19 podem contribuir para o desenvolvimento de sintomas ansiosos. As atividades laborativas, de lazer, a prática de atividade física e as práticas religiosas devem ser estimuladas, pois auxiliam a reduzir o risco de desenvolvimento de transtornos mentais.
\end{abstract}

Descritores: Prisioneiros. Infecções por Coronavírus. Saúde Mental. Mulheres. Ansiedade.

Objective: to investigate the self-report of anxiety symptoms related to Covid-19 among incarcerated women. Method: cross-sectional study, conducted in a prison unit in Salvador, Babia, from April to May 2020. Data were collected

\footnotetext{
Graduando em Enfermagem. Universidade do Estado da Bahia. Pesquisador do Núcleo de Pesquisa Interfaces em Saúde. Salvador, Bahia, Brasil. gcsantos.enf@gmail.com. http://orcid.org/0000-0003-2092-3753.

Graduanda em Enfermagem. Universidade do Estado da Bahia. Pesquisadora do Núcleo de Pesquisa Interfaces em Saúde. Salvador, Bahia, Brasil. http://orcid. org/0000-000 I-6874-9905.

Enfermeira. Pós-Doutora em Saúde Coletiva. Professora Adjunta do Colegiado de Enfermagem da Universidade do Estado da Bahia. Salvador, Bahia, Brasil. http:// orcid.org/0000-0002-5632-0973.

4 Enfermeira e Psicóloga. Pós-Doutora em Saúde Coletiva. Professora Associada I da Escola de Enfermagem da Universidade Federal da Bahia. Salvador, Bahia, Brasil. http://orcid.org/0000-0003-0295-9998.

5 Enfermeira. Doutora em Enfermagem. Professora Auxiliar do Colegiado de Enfermagem da Universidade do Estado da Bahia. Salvador, Bahia, Brasil. http://orcid. org/0000-0002-1963-0120.

6 Enfermeira. Mestra em Enfermagem. Professora Substituta da Escola de Enfermagem da Universidade Federal da Bahia. Salvador, Bahia, Brasil. http://orcid.org/00000002-8268-3380.
} 
through a questionnaire, and the relative and absolute frequencies were analyzed. Results: the participants were 41 women, mostly young, with low schooling, single mothers and black. 95\% of the sample reported some anxiety symptom. There was a bigh prevalence of concern with oneself, prison security and family before the pandemic. Most women have found mechanisms to mitigate the psychological impacts of the pandemic. Conclusion: the weakening of family ties and the feeling of insecurity related to Covid-19 may contribute to the development of anxious symptoms. Work activities, leisure activities, physical activity and religious practices should be encouraged, as they help reduce the risk of developing mental disorders.

Descriptors: Prisoners. Coronavirus Infections. Mental Health. Women. Anxiety.

Objetivo: investigar el auto-informe de los sintomas de ansiedad relacionados con Covid-19 entre las mujeres encarceladas. Método: estudio transversal, realizado en una unidad penitenciaria en Salvador, Bahia, de abril a mayo de 2020. Los datos fueron recogidos a través de un cuestionario y se analizaron las frecuencias relativas y absolutas. Resultados: Participaron 41 mujeres, en su mayoría jóvenes, con baja escolaridad, madres solas y negras. El 95\% de la muestra reportó algún síntoma de ansiedad. Se encontró una alta prevalencia de preocupación consigo, la seguridad penitenciaria y la familia frente a la pandemia. La mayoría de las mujeres han encontrado mecanismos para mitigar los impactos psicológicos de la pandemia. Conclusión: el debilitamiento de los lazos familiares y la sensación de inseguridad relacionada con Covid-19 pueden contribuir al desarrollo de sintomas ansiosos. Se deben fomentar las actividades de trabajo, las actividades de ocio, la actividad física y las prácticas religiosas, ya que ayudan a reducir el riesgo de desarrollar trastornos mentales.

Descriptores: Prisioneros. Infecciones por Coronavirus. Salud Mental. Mujeres. Ansiedad.

\section{Introdução}

A velocidade de propagação da recente pandemia provocada pelo SARS-CoV-2, mais conhecido como novo coronavírus (Covid-19), tornou necessária a adoção de medidas de controle sanitário, restrição da circulação de pessoas nos diversos ambientes e adoção de medidas de distanciamento social para conter a disseminação do vírus, a fim de reduzir os impactos no Sistema de Saúde e na mortalidade da população.

Dentre as orientações preconizadas pelo Ministério da Saúde (MS) do Brasil para a contenção do vírus, há a recomendação de higienizar frequentemente as mãos e os punhos, usar máscaras no ambiente extradomiciliar, adotar a etiqueta respiratória, manter o distanciamento de aproximadamente dois metros de qualquer pessoa com sintomas respiratórios, evitando o contato íntimo, higienizar objetos e superfícies, além de manter o ambiente limpo e bem ventilado $^{(1)}$.

Nesse contexto, o conhecido histórico de superlotação das unidades prisionais brasileiras, as limitações de acesso a ações e serviços de saúde, e a precariedade da estrutura física dessas unidades merecem atenção especial. No sistema penitenciário brasileiro as más condições de higiene, má-alimentação, sedentarismo e as celas úmidas e escuras são fatores que favorecem a proliferação de epidemias e o desenvolvimento de psicopatologias ${ }^{(2)}$.

Estima-se que, enquanto na população livre uma pessoa infectada contamina outras 2 a 3 pessoas, as condições do encarceramento propiciam que um infectado contamine até 10 pessoas. Sob essa análise, uma cela com 150 pessoas privadas de liberdade (PLL), dentro de 14 dias teria $67 \%$ de seus abrigados contaminados, e o seu total, em 21 dias $^{(3)}$.

Ademais, destaca-se a elevada prevalência de doenças como tuberculose, pneumonia, diabetes, hipertensão, câncer, pessoas vivendo com o vírus da imunodeficiência humana/síndrome da imunodeficiência adquirida (PVHIV/Aids), além da presença de idosos, gestantes e lactentes dentre as PPL, condições que colocam essas pessoas no grupo de maior risco para desenvolver sintomas graves da Covid-19 ${ }^{(3-4)}$.

A Lei de Execução Penal (LEP) e a Política Nacional de Atenção Integral à Saúde das Pessoas Privadas de Liberdade no Sistema Prisional (PNAISP) estabelecem a oferta de atendimento de caráter preventivo e curativo às PPL, bem 
como ações de promoção, proteção e recuperação da saúde. Em concordância, manual elaborado pelos Ministérios da Saúde e da Justiça e Segurança Pública ${ }^{(1)}$ recomenda que se mantenha a oferta de ações de saúde intramuros nesse período, acrescidas da observação de sintomas respiratórios e adoção de isolamento de casos suspeitos.

Entretanto, a permanência de condições insalubres, relações de violência, condições precárias de trabalho para as equipes de saúde prisional e a lógica punitiva do encarceramento destacam-se como fatores que dificultam a efetivação do direito à saúde para as $\mathrm{PPL}^{(5)}$.

Historicamente, os homens são mais associados a atos criminosos, representando o maior número de integrantes do sistema penitenciário brasileiro. A presença feminina nesses espaços é tida como fenômeno recente e crescente, ligada principalmente ao envolvimento com drogas ${ }^{(6)}$. No estado da Bahia, as mulheres representam aproximadamente $2,38 \%$ da população carcerária ${ }^{(7)}$, enquanto, no Brasil, correspondem a aproximadamente $4,94 \%{ }^{(8)}$.

É amplamente descrito na literatura, entretanto, que homens e mulheres vivem de modo diferente a experiência de encarceramento. Para a população feminina, a inserção em um sistema prisional, inicialmente criado por homens e para homens, favorece a negligência com os cuidados à saúde. Para além do não atendimento de necessidades típicas das mulheres, a experiência no cárcere aprofunda a marginalização social e as submetem a maiores riscos de vitimização por violência e adoecimento físico e mental ${ }^{(9)}$.

Em uma avaliação comparativa de depressão entre homens e mulheres encarcerados, a ocorrência de depressão mostrou-se cerca de duas vezes maior entre as mulheres, sendo identificado ainda que existem diferenças nos fatores associados à depressão entre homens e mulheres. A depressão entre mulheres mostra-se ainda como fator de risco para o surgimento de problemas físicos, sendo necessário, portanto, que se oferte diferentes estratégias de enfrentamento e adaptação para homens e mulheres ${ }^{(10)}$.
Em paralelo, a ação reguladora das prisões desenvolve um importante papel social e, ao mesmo tempo, determina o processo saúde-doença das PPL, uma vez que a prevalência de transtornos psíquicos entre mulheres privadas de liberdade é maior do que o adoecimento de mulheres em liberdade ${ }^{(11)}$, ratificando a urgência de que o sistema prisional assegure cuidados integrais e de caráter interdisciplinar.

Em maio de 2020, a Organização Mundial da Saúde (OMS) reconheceu que a pandemia da Covid-19 tem gerado grandes impactos na saúde mental dos diversos extratos sociais. Desvela, com isso, a necessidade de integrar o apoio à saúde mental como componente nas respostas de enfrentamento nacional à pandemia, considerando que sem saúde mental, não há saúde ${ }^{(12)}$.

Haja vista a urgência das medidas de contenção da pandemia e a mudança nos fluxos operacionais das instituições penitenciárias, o objetivo deste estudo é investigar o autorrelato de sintomas de ansiedade relacionados ao período pandêmico entre mulheres encarceradas em um centro prisional de Salvador, Bahia.

\section{Método}

Trata-se de estudo transversal, de caráter exploratório, com abordagem quantitativa, realizado no Conjunto Penal Feminino de Salvador, Bahia, no período de abril a maio de 2020.

As mulheres elegíveis para participar do estudo foram todas as que se encontravam em situação de privação de liberdade, residentes do local do estudo, e presentes no período de coleta dos dados. Foram excluídas do estudo as mulheres que gozaram da progressão de regime durante o período de realização da pesquisa.

Os dados foram coletados por meio da aplicação de um questionário anônimo e autoaplicável, para identificar o nível de informação sobre a transmissão e as medidas de prevenção contra o coronavírus, além de analisar a repercussão das medidas de distanciamento social em sua saúde mental. Este instrumento foi desenvolvido por membros do Núcleo de Pesquisa Interfaces 
em Saúde (NUPEIS) da Universidade do Estado da Bahia (UNEB).

Devido às recomendações de restrição de visitas nas unidades do sistema prisional em todo Brasil, e a fim de viabilizar a execução do estudo, os questionários e os termos de consentimento livre e esclarecido (TCLE) foram entregues à diretora do presídio, que designou a sua aplicação à psicóloga da equipe de saúde prisional, com apoio dos agentes prisionais. Assim, as mulheres foram convidadas a responder ao instrumento em uma sala, três participantes por vez, com o afastamento de 1,5 metro entre cada mulher e sob a supervisão da psicóloga da equipe de saúde prisional. Após a leitura e assinatura do TCLE, a coleta dos dados foi iniciada. Em caso de dificuldades com a leitura, dúvidas sobre as perguntas ou sobre a escolha das alternativas, as participantes receberam orientação da psicóloga.

Durante a pesquisa respeitou-se os princípios da autonomia, beneficência, não maleficência, justiça, liberdade, confidencialidade e equidade das participantes. Os TCLE estão sob a guarda do NUPEIS e serão mantidos sob os cuidados dos pesquisadores. As informações foram utilizadas apenas para fins acadêmicos.

As questões de múltipla escolha admitiram mais de uma possibilidade de resposta, as multirrespostas. Desse modo, em alguns casos, há mais de uma resposta considerada para a mesma questão.

Os dados coletados foram submetidos à análise descritiva, sendo organizados e processados no software Epi Info Windows versão Centers for Disease Control and Prevention (CDC). Os dados estão apresentados como frequências absolutas e relativas em tabelas, figura e gráfico.

Este estudo faz parte de um projeto denominado "O encarceramento e a saúde mental de mulheres em um presídio na cidade de Salvador BA", aprovado pelo Comitê de Ética em Pesquisa (CEP) da Universidade do Estado da Bahia sob Parecer $n^{\circ}$ 3.691.962.

\section{Resultados}

Participaram deste estudo 41 mulheres, com idade variando entre 20 e 57 anos, predominantemente solteiras (82,9\%), majoritariamente negras (pretas e pardas, 78,1\%), de baixa escolaridade, com tempo de reclusão variando entre 15 dias e 5 anos e 10 meses. A maioria das mulheres $(78,1 \%)$ possuem um ou mais filhos, ao passo que $21,9 \%$ não têm filhos. A caracterização das participantes encontra-se na Tabela 1.

Tabela 1 - Caracterização sociodemográfica da amostra de mulheres privadas de liberdade no Conjunto Penal Feminino de Salvador - 2020. (N=41)

(continua)

\begin{tabular}{l|c|c}
\hline Variáveis & $\mathbf{n}$ & $\mathbf{\%}$ \\
\hline Idade & & 58.5 \\
$20-29$ anos & 24 & 29.3 \\
$30-49$ anos & 12 & 7.3 \\
$\geq 50$ anos & 3 & 4.9 \\
Ignorado & 2 & \\
Estado civil & & 82.9 \\
Solteira & 34 & 4.9 \\
Casada & 2 & 4.9 \\
Viúva & 2 & 7.3 \\
União estável & 3 & \\
Raça/cor & & 19.5 \\
Branca & \multicolumn{2}{c}{} \\
Indígena & 8 & 2.4 \\
Parda & 1 & 33.9 \\
Preta & 18 & 34.2
\end{tabular}


Tabela 1 - Caracterização sociodemográfica da amostra de mulheres privadas de liberdade no Conjunto Penal Feminino de Salvador - 2020. (N=41)

(conclusão)

\begin{tabular}{l|c|c}
\hline Variáveis & $\mathbf{n}$ & $\mathbf{\%}$ \\
\hline Escolaridade & 1 & 2.4 \\
Analfabeta & 16 & 39 \\
Fundamental incompleto & 8 & 19.5 \\
Fundamental completo & 2 & 4.9 \\
Médio incompleto & 10 & 24.4 \\
Médio completo & 2 & 4.9 \\
Superior incompleto & 2 & 4.9 \\
Ignorado & & \\
Tempo de reclusão & 2 & 4.9 \\
$\leq 30$ dias & 22 & 53.7 \\
$>1$ mês $\leq 1$ ano & 10 & 24.4 \\
$>1$ ano $\leq 3$ anos & 4 & 9.7 \\
$>3$ anos & 3 & 7.3 \\
Ignorado & & \\
Filhos & 9 & 21.9 \\
0 & 21 & 51.2 \\
1 até 2 filhos & 11 & 26.9 \\
\hline 2 filhos & & \\
\hline
\end{tabular}

Fonte: Elaboração própria.

Em acordo à recomendação do Conselho Nacional de Justiça no 62/2020, que orienta medidas de prevenção à propagação da Covid-19 no âmbito dos sistemas de justiça penal e socioeducativo, 43 mulheres privadas de liberdade deste Conjunto Penal foram beneficiadas com a progressão de regime, passando a cumprir sua pena em ambiente domiciliar, enquanto durar a pandemia, devido à presença de comorbidades que as predispõem ao desenvolvimento de formas graves da Covid-19.

Quando questionadas sobre a repercussão da diminuição do fluxo de pessoas na unidade prisional, as respostas variaram entre "não mudou minha rotina", "me sinto insegura aqui", "sinto falta das visitas" e "tenho me sentido muito preocupada ou ansiosa". A representação dessas respostas encontra-se ilustrada na Figura 1. 
Figura 1 - Avaliação da restrição da circulação de pessoas no Conjunto Penal Feminino de Salvador durante o período de distanciamento social

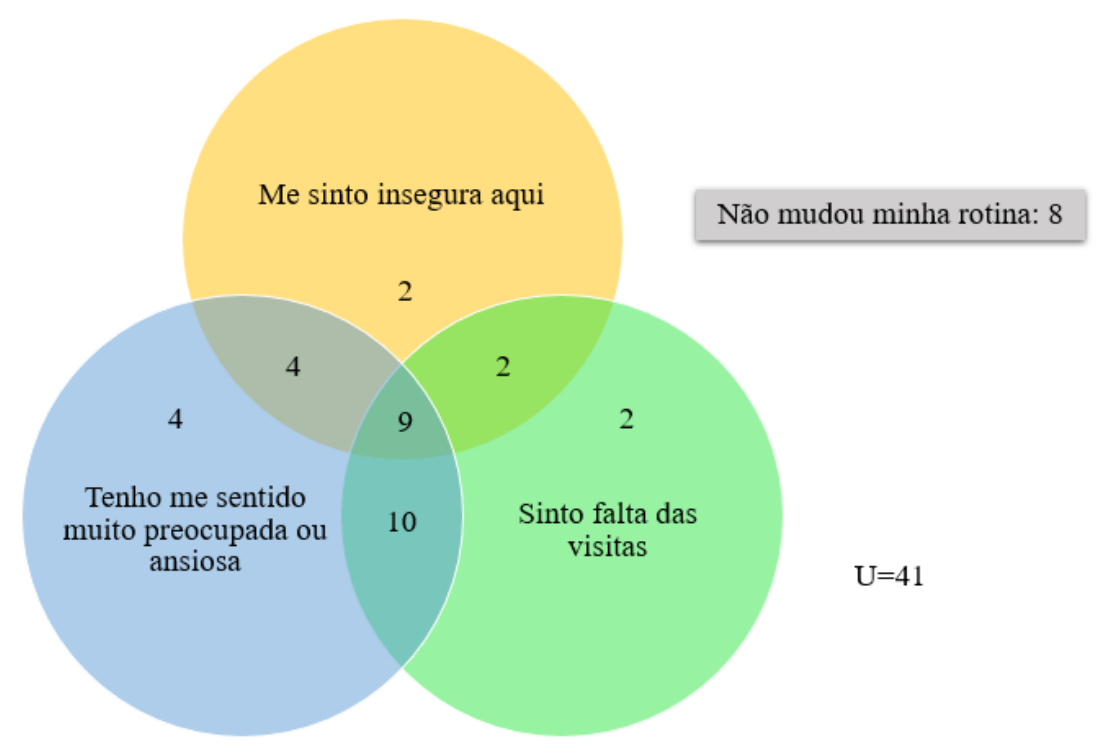

Fonte: Elaboração própria.

A avaliação dos sintomas decorrentes do distanciamento social está representada na Tabela
2. Destaca-se que $95,1 \%$ das mulheres experimentam algum sintoma ansioso.

Tabela 2 - Frequência de autorrelato de sintomas ansiosos decorrentes do distanciamento social na amostra de mulheres privadas de liberdade no Conjunto Penal Feminino de Salvador -2020 . (N=41) (continua)

\begin{tabular}{|c|c|c|}
\hline Variáveis & $\mathbf{f r} \mathbf{r}^{(1)}$ & fr $\%$ \\
\hline \multicolumn{3}{|l|}{ Alterações sensoriais, motoras e do equilíbrio } \\
\hline Dormência ou formigamento & $15 / 41$ & 36.6 \\
\hline Sensação de calor & $4 / 41$ & 9.8 \\
\hline Tremores nas pernas & $3 / 41$ & 7.3 \\
\hline Perda do equilíbrio & $7 / 41$ & 17.1 \\
\hline Tremores nas mãos & $6 / 41$ & 14.6 \\
\hline Sensação de desmaio & $5 / 41$ & 12.2 \\
\hline Suor (não devido ao calor) & $5 / 41$ & 12.2 \\
\hline \multicolumn{3}{|l|}{ Alterações do humor e comportamento } \\
\hline Medo de que aconteça o pior & $33 / 41$ & 83.5 \\
\hline Aterrorizado & $13 / 41$ & 33.7 \\
\hline Nervoso & $22 / 41$ & 52.7 \\
\hline Medo de perder o controle & $16 / 41$ & 46.3 \\
\hline Medo de morrer & $12 / 41$ & 29.3 \\
\hline Preocupação com problemas físicos & $12 / 41$ & 29.3 \\
\hline Tristeza além do que sentia antes da quarentena & $32 / 41$ & 78 \\
\hline
\end{tabular}


Tabela 2 - Frequência de autorrelato de sintomas ansiosos decorrentes do distanciamento social na amostra de mulheres privadas de liberdade no Conjunto Penal Feminino de Salvador $-2020 .(\mathrm{N}=41)$ (conclusão)

\begin{tabular}{|c|c|c|}
\hline Variáveis & $\mathbf{f r}^{(1)}$ & fr $\%{ }^{(2)}$ \\
\hline \multicolumn{3}{|l|}{ Alterações do humor e comportamento } \\
\hline Desânimo para realizar atividades cotidianas & $22 / 41$ & 53.7 \\
\hline Choro além do que chorava antes da quarentena & $27 / 41$ & 65.8 \\
\hline Diminuição do prazer em relação à vida & $11 / 41$ & 28.8 \\
\hline Perda do otimismo em relação ao futuro & $13 / 41$ & 31.7 \\
\hline Aumento da irritabilidade & $18 / 41$ & 43.9 \\
\hline $\begin{array}{l}\text { Sente mais dificuldade em tomar decisões ou começar alguma } \\
\text { atividade agora do que antes da quarentena }\end{array}$ & $10 / 41$ & 24.4 \\
\hline Perdeu o interesse em estar com as pessoas & $6 / 41$ & 14.6 \\
\hline Sente-se menos otimista em relação ao futuro & $13 / 41$ & 31.7 \\
\hline \multicolumn{3}{|l|}{ Alterações do sono e repouso } \\
\hline Incapaz de relaxar & $25 / 41$ & 61 \\
\hline Cansaço e indisposição & $10 / 41$ & 24.4 \\
\hline Piora na qualidade do sono & $27 / 41$ & 65.8 \\
\hline \multicolumn{3}{|l|}{ Alterações cardiorrespiratórias } \\
\hline Palpitação ou aceleração do coração & $15 / 41$ & 36.6 \\
\hline Dificuldade de respirar & $4 / 41$ & $9.8 \%$ \\
\hline Sensação de sufocação & $6 / 41$ & 14.6 \\
\hline \multicolumn{3}{|l|}{ Alterações gastrointestinais } \\
\hline Sensação de "borboletas" no estômago & $4 / 41$ & 9.8 \\
\hline Náusea/enjoo & $3 / 41$ & 7.3 \\
\hline Indigestão ou desconforto no abdômen & $10 / 41$ & 24.4 \\
\hline Alteração do apetite & $16 / 41$ & 39 \\
\hline Não respondeu & $2 / 41$ & 4.9 \\
\hline
\end{tabular}

Fonte: Elaboração própria.

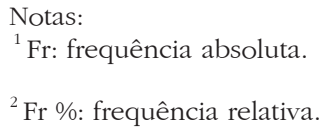

Ao serem perguntadas sobre a frequência em que pensa na situação do país em relação à pandemia do novo coronavírus, 9,8\% referiram não pensar ou não se preocupar; $4,9 \%$ relataram pensar algumas vezes, mas não se preocupar; $29,3 \%$ pensam e se preocupam algumas vezes; $43,9 \%$ pensam e se preocupam a maior parte do tempo; e 12,2\% não responderam.

Já em relação à situação da família no contexto da pandemia, 70,8\% pensam e se preocupam a maior parte do tempo; $19,5 \%$ pensam e se preocupam algumas vezes; $4,9 \%$ pensam, mas não se preocupam; 2,4\% não pensam ou não se preocupam com a família neste momento; e 2,4\% não responderam.

$\mathrm{Na}$ avaliação das estratégias adotadas para manutenção da saúde mental neste período, $39,0 \%$ das mulheres relataram realizar atividades laborativas; 48,78\% praticam atividades de lazer; $12,2 \%$ se valem da prática de atividade física; $17,1 \%$ apontam as práticas religiosas e espirituais como estratégia; 9,8\% citam o diálogo; $2,4 \%$ pensam na família; 4,9\% dormem; $2,4 \%$ relataram ficar sozinha; e 7,3\% referiram não adotar nenhuma estratégia para manter a saúde mental (Gráfico 1). 
Gráfico 1 - Estratégias adotadas por mulheres privadas de liberdade no Conjunto Penal Feminino de Salvador para manter a saúde mental durante o período de isolamento social

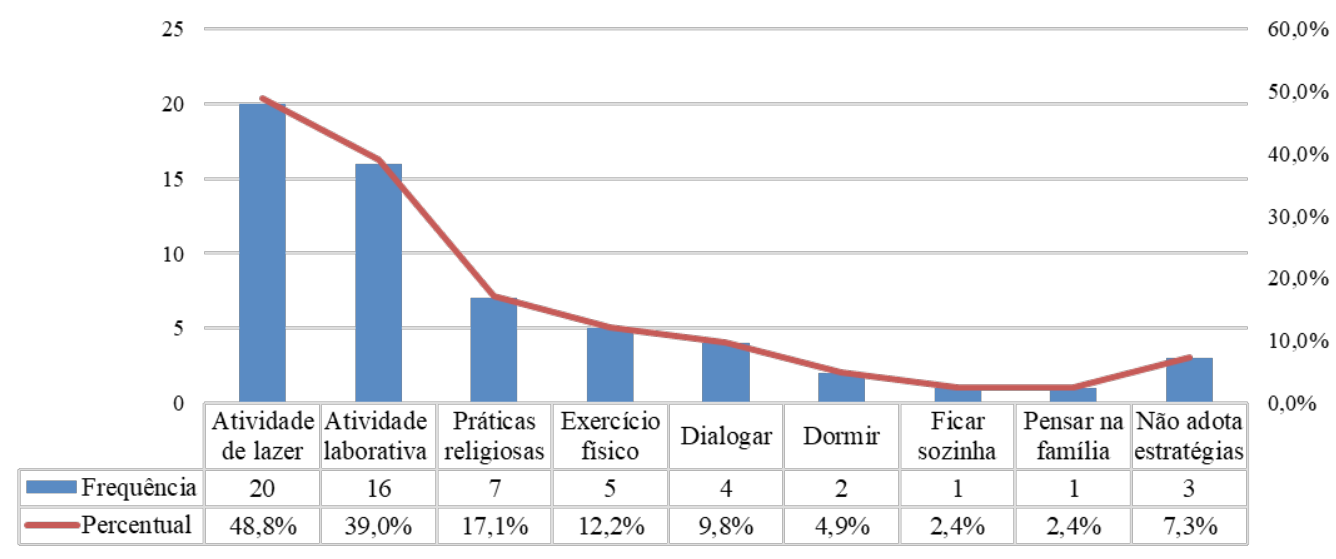

Fonte: Elaboração própria.

Quanto à necessidade de ajuda profissional para reduzir as preocupações nesse momento de pandemia, 51,2\% referiram não precisar; 46,3\% relataram necessitar de ajuda; e 2,4\% não souberam dizer.

\section{Discussão}

De modo semelhante aos resultados encontrados em outros estudos nacionais, encontrou-se nesta amostra a prevalência de mulheres jovens, negras, solteiras, de baixa escolaridade e que possuem pelo menos um filho.

Destaca-se que a raça/cor de uma população reflete fatores socioculturais, vinculados a contextos regionais e da colonização do país. Como espelho, o perfil das mulheres em estudo realizado em um presídio no estado de São Paulo, encontrou a predominância de mulheres brancas em privação de liberdade ${ }^{(13)}$. Tendo em vista a predominância da raça negra na cidade de Salvador, os dados encontrados refletem meramente a constituição dessa população, não devendo a raça negra ser vinculada a atos de criminalidade e, portanto, do seu encarceramento.

A predominância de mulheres solteiras no Conjunto Penal estudado é um fenômeno debatido pela literatura, ao reconhecer que a interrupção dos laços familiares nas unidades prisionais femininas é um fato comum e que diverge da realidade de unidades penais masculinas, sendo a perda do companheiro durante o período de encarceramento vista por essas mulheres como "destino" (14).

Nessa perspectiva, a condição de mãe solo é descrita como produto do envolvimento dos pais com a criminalidade, de modo que muitos se encontram presos ou foragidos, além daqueles que não reconheceram a paternidade de seus filhos. Às crianças resta o cuidado dos familiares, principalmente dos avós maternos, ou de abrigos de doação, o que torna a experiência de encarceramento ainda mais difícil, tendo em vista a fragilização da relação mãe-filho ${ }^{(6,9,14)}$.

Indo além, a potência do ambiente prisional como determinante social da saúde mental contribui para o surgimento de sintomas e comportamentos temporários ou permanentes. Isso se justifica pelas mudanças bruscas que catalisam estressores e potencializam sensações relacionadas à ansiedade e depressão ${ }^{(11)}$.

Tendo em vista a restrição da circulação de pessoas no Sistema Prisional, bem como a liberdade concedida a companheiras de cárcere, em função da pandemia, como uma mudança repentina na rotina das mulheres privadas de liberdade, justifica-se, assim, o relato de maior ansiedade e preocupação entre 27 (65,8\%) mulheres deste estudo, bem como o relato de 95,1\% da população estudada de pelo menos um sintoma ansioso no período. 
A alta prevalência de queixas relacionadas a alterações de humor e comportamento encontradas neste estudo pode ser explicada por duas hipóteses. A primeira, defende que estas são as alterações mais precoces e comumente percebidas em crises de ansiedade. Outra possível explicação baseia-se na frequência em que essas mulheres relataram pensar e se preocupar com a situação do país e da família no contexto da pandemia, sobretudo quando se considera maior fragilização dos vínculos sociais e familiares.

É nessa esteira que a suspensão do contato com a família intensifica a sensação de isolamento e insegurança, aumentando a preocupação com a própria vida e com os familiares. Dentre o conjunto de estratégias para minimizar tal impacto, o acesso à informação para a proteção, prevenção e assistência à saúde, além da viabilização da comunicação com os familiares por meio de cartas, ligações e outros meios disponíveis para este fim $^{(3)}$, são descritos como elementos atenuantes do processo de encarceramento.

Em seguimento, percebe-se que as estratégias adotadas para a manutenção da saúde mental durante a pandemia concordam com práticas já realizadas antes desse período, como apontam os estudos que descrevem as atividades laborativas, as visitas dos familiares, a prática religiosa, maior tempo de reclusão e a expectativa de retornar ao convívio familiar e social como fatores de proteção ao sofrimento mental entre as $\operatorname{PPL}^{(6,11,14-15)}$.

Chama a atenção a realização de práticas de lazer como a principal estratégia de cuidado com a saúde mental, elemento pouco citado na literatura. Neste estudo, 48,8\% das mulheres referiram atividades, como leitura (não religiosa), ouvir música, assistir televisão e os jogos de tabuleiro, como ferramentas utilizadas para reduzir o estresse psicológico durante a pandemia, superando a utilização de outras estratégias já consagradas pela literatura. Essa observação alerta para novas formas de reduzir o sofrimento mental durante o encarceramento, sendo uma alternativa de fácil implementação e baixo custo.

Embora a presença de sintomas ansiosos tenha sido relatada por quase todas as mulheres deste estudo, apenas 46,3\% destas mulheres referiram sentir a necessidade de ajuda ou acompanhamento profissional. Isso pode se explicar pela constante busca de estratégias de adaptação aos desafios da institucionalização prisional que culmina no surgimento de elementos que denotam o desenvolvimento de traços de resiliência perante as adversidades da prisão ${ }^{(14)}$.

Fatores associados à identificação de transtornos mentais comuns entre mulheres encarceradas têm associação com o histórico pregresso, a exemplo de baixa renda, uso de drogas, ter testemunhado violência na infância/adolescência e ter sido vítima de violência no ano anterior ao da prisão ${ }^{(16)}$. Apesar de serem condições que agravam o risco para transtornos mentais, pode ter havido influência nas respostas, por não serem condições ligadas diretamente a este momento.

Outrossim, a hierarquia imposta no sistema prisional, bem como as constantes violações de direito nesses ambientes, alteram a percepção do direito à saúde entre as PPL, quer pela necessidade de submeter-se constantemente à ordem disciplinar para ter efetivado o seu direito, quer pelas precárias condições de trabalho dos profissionais de saúde nesses espaços; as PPL, por vezes, sentem-se destituídas do direito à saúde, enquanto encarceradas ${ }^{(5)}$.

A restrição de circulação na unidade prisional e, por conseguinte, a diminuição da quantidade de aplicadores do questionário, nos fez optar apenas pela utilização de frequência de autorrelatos de sintomas ansiosos, não tendo sido mensurada a sua intensidade, impossibilitando a realização de análises mais complexas, o que configura uma limitação deste estudo.

Assim, este estudo aponta para a necessidade de reconhecer que, para além das medidas de prevenção da propagação da Covid-19 nos ambientes prisionais, é preciso aumentar a vigilância e os cuidados com a saúde mental da população carcerária nesse período, integrando estratégias de fortalecimento dos vínculos familiares e sociais, mediante tecnologias e estratégias de integração desenvolvidas internamente. 


\section{Conclusão}

Os dados desta pesquisa incorporam o escopo já debatido na literatura, que reconhece a predominância de mulheres jovens, de baixa escolaridade e mães solo em instituições prisionais, fatores que vulnerabilizam esse estrato social à marginalização, à desigualdade de oportunidades, ao envolvimento com atos infracionais e, consequentemente, com o encarceramento.

Percebeu-se elevada prevalência de sintomas ansiosos relacionados com a implementação de medidas mais restritivas no ambiente prisional, em decorrência da pandemia do coronavírus. Embora essas mulheres vivenciem no seu cotidiano experiências que comprometem a saúde mental, a presença de uma ameaça física e invisível, além da proibição de visitas, neste momento, gera sensação ainda maior de insegurança, ansiedade e preocupação.

Nesse campo, cabe à equipe de saúde prisional, que conta com profissionais médicos, enfermeiros, psicólogos e assistentes sociais, desenvolver ações educativas, protetivas, preventivas, de vigilância e de promoção da saúde, tanto na formulação de protocolos internos para contenção da Covid-19 quanto em atenção à saúde mental, uma vez que a pandemia evoca ações sensíveis para garantir a segurança das mulheres privadas de liberdade.

Ademais, a manutenção das atividades laborativas, recreativas, religiosas e educativas precisa ser estimulada, dentro das possibilidades de segurança biológica, uma vez que se configuram elementos protetores para a saúde mental nesse contexto.

Por fim, reforça-se a necessidade de expandir as discussões sobre os direitos de pessoas privadas de liberdade, tendo em vista a necessidade da construção de novos modelos de ressocialização capazes de interligar justiça, saúde, educação e cidadania, de modo que se alcance o ideal de instituição ressocializadora, com o mínimo de prejuízo à saúde física e mental das reeducandas.

\section{Contribuições:}

1 - concepção, projeto, análise e interpretação dos dados: Gabriel da Cruz Santos, Tainá Cerqueira Simôa, Tânia Christiane Ferreira Bispo, Ridalva Dias Martins e Aglaya Oliveira Lima Cordeiro de Almeida;

2 - redação do artigo e revisão crítica relevante do conteúdo intelectual: Gabriel da Cruz Santos, Tainá Cerqueira Simôa, Tânia Christiane Ferreira Bispo, Ridalva Dias Martins, Denise Santana Silva dos Santos e Aglaya Oliveira Lima Cordeiro de Almeida;

3 - aprovação final da versão a ser publicada: Gabriel da Cruz Santos, Tainá Cerqueira Simôa, Tânia Christiane Ferreira Bispo, Ridalva Dias Martins, Denise Santana Silva dos Santos e Aglaya Oliveira Lima Cordeiro de Almeida.

\section{Referências}

1. Brasil. Ministério da Saúde. Ministério da Justiça e Segurança Pública. Departamento Penitenciário Nacional. Manual recomendações para prevenção e cuidado da Covid-19 no Sistema Prisional brasileiro [Internet]. Brasília (DF); 2020 [cited 2020 May 5]. Available from: https://www.gov.br/ depen/pt-br/ManualCOVID19DEPEN1edicao.pdf/ view

2. Soares Filho MM, Bueno PMMG. Demografia, vulnerabilidades e direito à saúde da população prisional brasileira. Ciên saúde coletiva. 2016 jul;21(7):1999-2010. DOI: https://doi.org/ 10.1590/1413-81232015217.24102015

3. Sánchez A, Simas L, Diuana V, Larouze B. COVID-19 nas prisões: um desafio impossível para a saúde pública? Cad Saúde Pública. 2020;36(5):e00083520. DOI: https://doi.org/10.1590/0102-311x00083520

4. Souza AV, Rodrigues TTMB. O Direito à Saúde da População em Privação de Liberdade Frente às Medidas de Contenção do coronavírus (Covid-19) [Internet]. Salvador; 2020 [cited 2020 May 3]. Available from: https://ccrim.ufba. br/o-direito-saude-da-populacao-em-privacaode-liberdade-frente-medidas-de-contencao-docoronavirus

5. Martins ÉLC, Martins LG, Silveira AM, Melo EM. O contraditório direito à saúde de pessoas em 
privação de liberdade: o caso de uma unidade prisional de Minas Gerais. Saúde soc. 2014 Dec;23(4):1222-34. DOI: https://doi.org/10.1590/ S0104-12902014000400009

6. Neri MS, Oliveira JF, Nascimento ER, Gusmão MEN, Moreira VS. Presas pelas drogas: características de saúde de presidiárias em Salvador, Bahia. Rev baiana enferm [Internet]. 2011 [cited 2020 Jul 6];121-32. Available from: https://portalseer. ufba.br/index.php/enfermagem/article/view/ $5908 / 4465$

7. Bahia. Secretaria de Administração Penitenciária e Ressocialização. Central de Informação e Documentação. Mapa da população carcerária do Estado da Bahia (por regimes) [Internet]. Salvador; 2020 [cited 2020 Oct 15]. Available from: http:// www.seap.ba.gov.br/pt-br/dados/

8. Brasil. Ministério da Justiça e Segurança Pública. Departamento Penitenciário Nacional. Levantamento Nacional de Informações Penitenciárias [Internet]. Brasília (DF); 2019 [cited 2020 Jul 11] Available from: https://app.powerbi. com/w?r=eyJrljoiZTlkZGJjODQtNmJlMi00OTJhLW FlMDktNzRlNmFkNTM0MWI3IiwidCI6ImViMDkw NDIwLTQ0NGMtNDNmNy05MWYyLTRiOGRhNm JmZThlMSJ9

9. Isaac FF, Campos TPR. O Encarceramento Feminino no Brasil [Internet]. Rio de Janeiro: Fundação Osvaldo Cruz; 2019 [cited 2020 Jul 12]. Available from: https://cee.fiocruz.br/?q=node/997

10. Santos MM, Barros CRS, Andreoli SB. Fatores associados à depressão em homens e mulheres presos. Rev bras epidemiol. 2019;22:e190051. DOI: https://doi.org/10.1590/1980-549720190051

11. Santos MV, Alves VH, Pereira AV, Rodrigues DP, Marchiori GRS, Guerra JVV. Saúde mental de mulheres encarceradas em um presídio do Estado do Rio de Janeiro. Texto contexto - enferm. 2017;26(2):1-10. DOI: https://doi. org/10.1590/0104-07072017005980015

12. Organização Mundial da Saúde. "O impacto da pandemia na saúde mental das pessoas já é extremamente preocupante" [Internet]. Genebra (CHE); 2020 [cited 2020 Jul 13]. Available from: https://www.youtube.com/watch?time_ continue $=136 \& v=q s j$ q 4 Pws 2 I\&feature $=$ emb_logo

13. Moraes PAC, Dalgalarrondo P. Mulheres encarceradas em São Paulo: saúde mental e religiosidade. J bras psiquiatr. 2006;55(1):50-6. DOI: https://doi. org/10.1590/S0047-20852006000100007

14. Lima GMB, Pereira Neto AF, Amarante PDC, Dias MD, Ferreira Filha MO. Mulheres no cárcere: significados e práticas cotidianas de enfrentamento com ênfase na resiliência. Saúde debate. 2013 Set;37(98):446-56. DOI: http://dx.doi.org/10.1590/ S0103-11042013000300008

15. Canazaro D, Argimon IIL. Características, sintomas depressivos e fatores associados em mulheres encarceradas no Estado do Rio Grande do Sul, Brasil. Cad Saúde Pública. 2010;26(7):1323-33. DOI: https://doi.org/10.1590/ S0102-311X2010000700011

16. Audi CAF, Santiago SM, Andrade MGG, Francisco PMSB. Common mental disorder among incarcerated women: a study on prevalence and associated factors. Ciênc saúde coletiva. 2018 nov;23(11):3587-96. DOI: https://doi. org/10.1590/1413-812320182311.30372016

Recebido: 4 de agosto de 2020 Aprovado: 7 de outubro de 2020

Publicado: 26 de outubro de 2020

A Revista Baiana de Enfermagem utiliza a Licença Creative Commons - Atribuição-NãoComercial 4.0 Internacional. https://creativecommons.org/licenses/by-nc/4.0/

Este artigo é de acesso aberto distribuído sob os termos da Licença Creative Commons (CC BY-NC). Esta licença permite que outros remixem, adaptem e criem a partir do seu trabalho para fins não comerciais. Embora os novos trabalhos tenham de lhe atribuir o devido crédito e não possam ser usados para fins comerciais, os usuários não têm de licenciar esses trabalhos derivados sob os mesmos termos. 